\title{
Vortex flow in the left atrium in healthy controls and patients with mitral valve regurgitation after atrioventricular septal defect correction: evaluation with 4D Flow MRI and particle tracing
}

Emmeline Calkoen ${ }^{1 *}$, Patrick J de Koning ${ }^{2}$, Rob J van der Geest ${ }^{2}$, Albert de Roos ${ }^{2}$, Jos J Westenberg ${ }^{2}$, Arno Roest ${ }^{1}$

From 18th Annual SCMR Scientific Sessions

Nice, France. 4-7 February 2015

\section{Background}

During systole the left atrium serves as a reservoir, in which the inflow from the pulmonary veins is collected and partially organized by vortical flow [Fyrenius et al. Heart, 2001]. Aim of current study was to depict the origin of this vortical flow and evaluate the impact of mitral valve regurgitation on this flow structure.

\section{Methods}

12 healthy controls (age 9-53 years) and 8 patients (age 8-37 years) with a corrected atrioventricular septal defect and a mean mitral valve regurgitation of $25 \%$ (range 19$37 \%)$ were included. The maximal left atrial volume (LAV) was calculated based on the biplane area-length method from a standardized 2- and 4-chamber view. Whole-heart 4D Flow MRI was performed on a 3T MR scanner with free breathing, three-directional velocity encoding of $150 \mathrm{~cm} / \mathrm{s}$ in all directions, spatial resolution $2.3 \times 2.3 \times 3.0-4.2 \mathrm{~mm}^{3}$ and 30 phases reconstructed over one cardiac cycle. At end-systole the vortical flow was assessed by manually segmenting the volume of circular flow in the left atrium based on streamline visualization in a stack of slices parallel to the 4-chamber view. 3Dparticle tracing was applied in reverse time order, using the defined volume as seeding, to trace back the seed points and quantify the number of particles originating from the left and right pulmonary veins (LPV and RPV).

1Pediatric Cardiology, Leiden University Medical Center, Leiden, Netherlands Full list of author information is available at the end of the article

\section{Results}

In controls mean LAV was $60 \pm 35 \mathrm{~mL}$ and the vortex flow volume at end-systole $8 \pm 5 \mathrm{~mL}$. Tracing revealed a dominating contribution to the vortical volume originating from the LPV $(41 \pm 14 \%)$, a smaller part from the RPV $(17 \pm 12 \%)$ and a residual part of particles present inside the atrium at the start of systole $(42 \pm 15 \%)$.

In patients with mitral regurgitation the LAV was 76 $\pm 21 \mathrm{~mL}$ and a complex shape of the vortical flow with variation between subjects was observed (Figure 1). Mean vortical volume at end-systole was $5 \pm 4 \mathrm{~mL}$, with $13 \pm 14 \%$ contribution from LPV (difference with controls, $\mathrm{p}=0.001$ ), $6 \pm 6 \%$ from the RPV $(\mathrm{p}=0.049)$ and a residual part of 81 $\pm 14 \%$ (particles already inside the atrium at start of systole or coming from the ventricle as regurgitation) $(\mathrm{p}<0.001)$. Mean contribution ratio between LPV versus RPV was variable including patients with solely contribution from RPV or LPV.

\section{Conclusions}

In normal subjects, the vortical flow inside the left atrium during systole originates from both LPV and RPV, with a higher contribution from the left side. Mitral valve regurgitation disturbs this organized flow, resulting in a reduced contribution of LPV to the vortical flow, potentially leading to less efficient ventricular filling and stasis.

\section{Funding}

E.E. Calkoen is financially supported by a grant from the Willem-Alexander Kinder- en Jeugdfonds, J.J.M. Westenberg is financially supported by a grant from the Dutch Technology Foundation (STW), project number 11626. 

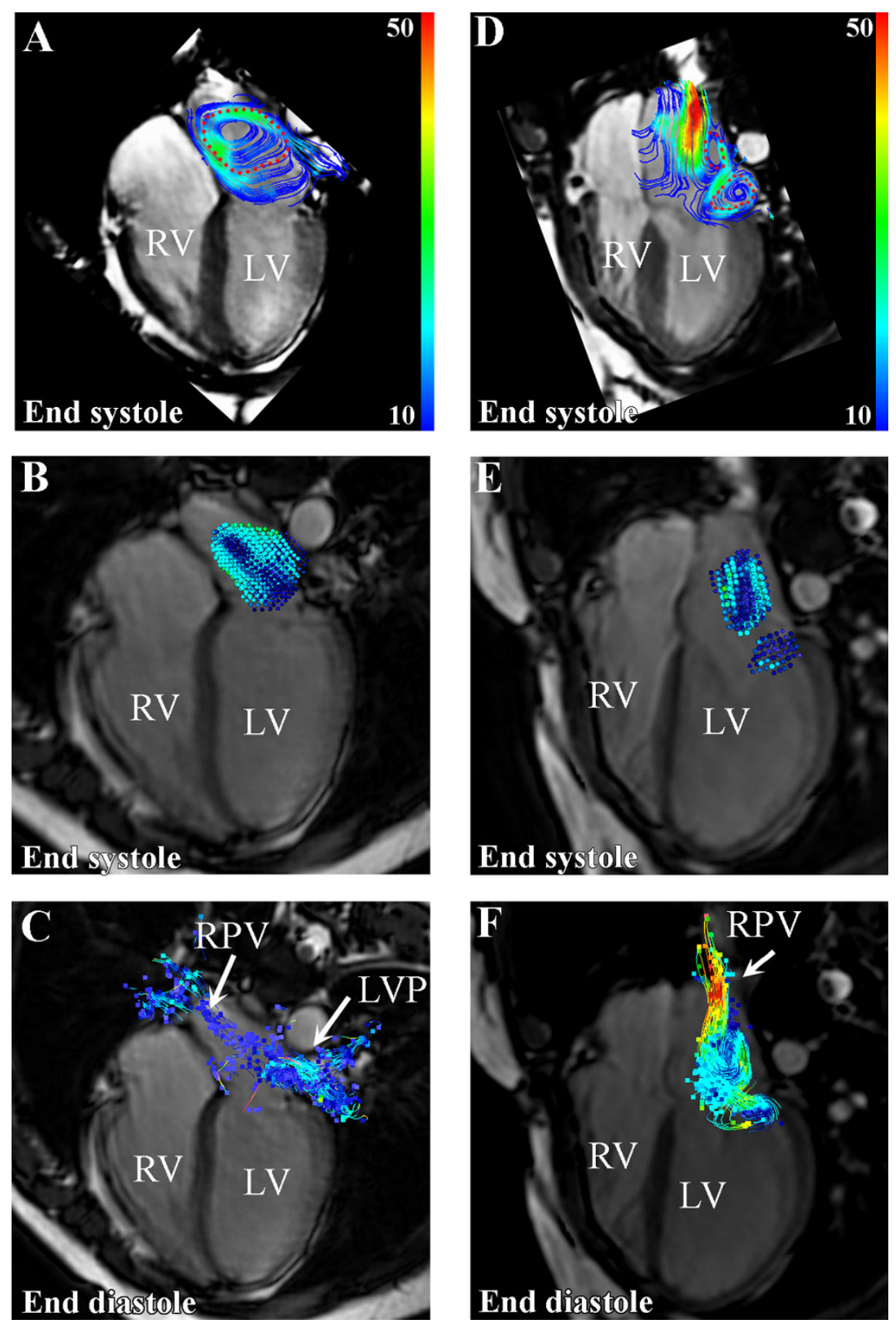

Figure 1 Vortical flow in the left atrium in a control subject $(A-C)$ and patient with laterally directed regurgitation (D-F). In $A$ and $D$ the red dotted contour indicate the segmented region of vortical flow. B and E shows the particle distribution at end-systole. $\mathrm{C}$ and $\mathrm{F}$ depicts the origin of the segmented vortical flow region as assessed by backward particle tracing.

\section{Authors' details}

${ }^{1}$ Pediatric Cardiology, Leiden University Medical Center, Leiden, Netherlands.

${ }^{2}$ Radiology, Leiden University Medical Center, Leiden, Netherlands.

Published: 3 February 2015
doi:10.1186/1532-429X-17-S1-0123

Cite this article as: Calkoen et al:: Vortex flow in the left atrium in healthy controls and patients with mitral valve regurgitation after atrioventricular septal defect correction: evaluation with 4D Flow MRI and particle tracing. Journal of Cardiovascular Magnetic Resonance 201517 (Suppl 1):Q123. 